\title{
Divergence insufficiency alleviated by posterior fossa decompression with duraplasty in a patient with Chiari type 1.5 malformation
}

\author{
Allison Strickland, MD, ${ }^{1}$ Cordell M. Baker, MD, ${ }^{1}$ R. Michael Siatkowski, MD, ${ }^{2}$ and \\ Timothy B. Mapstone, MD' \\ 1Department of Neurosurgery, University of Oklahoma Health Sciences Center; and 2Department of Ophthalmology, Dean \\ McGee Eye Institute, University of Oklahoma College of Medicine, Oklahoma City, Oklahoma
}

\begin{abstract}
The authors present a case of Chiari type 1.5 malformation with the uncommon presenting symptoms of esotropia and diplopia due to divergence insufficiency in a 12-year-old girl. Imaging at initial diagnosis revealed cerebellar herniation with extension of the tonsils to the $\mathrm{C} 2$ vertebral body, a retroflexed odontoid, and a small cervical syrinx. The patient was initially treated with an uncomplicated Chiari malformation decompression without dural opening. Repeat imaging revealed an adequate decompression. Three months postoperatively the patient's diplopia recurred and she underwent repeat posterior fossa decompression with dural opening and duraplasty. Following repeat decompression with dural opening and duraplasty, the patient's diplopia had not recurred by the 2-year follow-up.
\end{abstract}

https://thejns.org/doi/abs/10.3171/2018.5.PEDS1886

KEYWORDS Chiari 1.5 malformation; decompression; diplopia; divergence insufficiency; duraplasty

$\mathrm{C}$ HIARI malformation is a hindbrain abnormality of the posterior fossa in which the cerebellar tonsils herniate through the foramen magnum. The diagnostic criterion that is often cited is tonsillar herniation greater than $5 \mathrm{~mm}$ below the level of the foramen magnum. ${ }^{1}$ The abnormality is thought to result from compression of neural structures in the posterior fossa and is often associated with a spinal cord syrinx or skeletal abnormalities such as basilar impression, a retroflexed odontoid process, atlantooccipital fusion, atlantoaxial assimilation, and Klippel-Feil syndrome..$^{19}$ Common symptoms at presentation are headache, neck pain, weakness, numbness, loss of temperature sensation, and unsteadiness..$^{14}$ Less common symptoms include diplopia, esotropia, dysphasia, tinnitus, vomiting, and dysarthria. ${ }^{6,9,12,14,15}$ Posterior fossa decompression, with or without dural opening, has been shown to improve or alleviate symptoms. ${ }^{3,9,10}$

An infrequent presentation of Chiari malformation is diplopia due to divergence insufficiency. Most childhood esotropias present with a larger deviation for near than distance viewing; in divergence insufficiency, the opposite occurs. Patients with divergence insufficiency experience double vision when viewing distant objects but not when viewing objects near. ${ }^{8}$ In this case, symptoms in a patient with Chiari type 1.5 malformation, esotropia, and divergence insufficiency were corrected by posterior fossa decompression with dural opening and duraplasty.

\section{Case Report}

A 12-year-old girl developed new-onset binocular horizontal diplopia in the fall of 2013. Examination by ophthalmology revealed 20/20 visual acuity in each eye and normal anterior and posterior segments. There was a 12-prism diopter (PD) comitant esotropia at distance with full versions; near measurements were not recorded. In February 2014, she underwent a $3.5-\mathrm{mm}$ recession of the right medial rectus muscle. Immediately postoperatively she was seeing single, but within 2 weeks the diplopia recurred. At 3 weeks postoperatively, she was measured to 
have a comitant 12-PD esotropia at distance again, and was orthophoric at near. MRI of the brain revealed a Chiari 1.5 malformation with cerebellar herniation extending to the $\mathrm{C} 2$ vertebral body, craniocervical kyphosis, and significant brainstem compression. She also had a retroflexed odontoid process and small cervical syrinx (Fig. 1).

The patient presented to the ophthalmology clinic 2 months after recession of the right medial rectus muscle, with visual acuity of 20/20 in each eye and normal anterior and posterior segment exams. There was a comitant 20-PD esotropia at distance and an intermittent esotropia of 12 PDs at near. Near stereoacuity was 40 arcseconds. Cycloplegic retinoscopy was $+1.00+0.25 \times 90$ of the right eye, and $+0.75+0.25 \times 90$ of the left eye. In the late spring of 2014, the patient underwent an uncomplicated Chiari malformation decompression without dural opening. A $3-\mathrm{cm}$ by $3-\mathrm{cm}$ area of bone was removed above the foramen magnum with a $\mathrm{Cl}$ laminectomy, and constricting bands overlying the dura were lysed to decompress the tonsils and to allow adequate flow of CSF. There appeared to be good CSF pulsations under the dura, and a duraplasty did not appear to be warranted at this time. One month later, examination revealed a comitant esotropia of 20-25 PDs at distance and near along with a 3-PD comitant right hypertropia, felt to represent a skew deviation. Examination showed stable results 10 weeks later, and the patient underwent $4.5-\mathrm{mm}$ recession of the left medial rectus and a $7.0-\mathrm{mm}$ resection of the left lateral rectus with $3-\mathrm{mm}$ superior transposition. Examination 1 day postoperatively showed an intermittent esotropia of 2 PDs at distance and orthophoria at near. There was no diplopia. Examination 1 week postoperatively was stable, but two weeks later the patient reported recurrent diplopia and she had a comitant, constant esotropia of 15 PDs at distance and an intermittent esotropia of 12 PDs at near. There was no evidence of a slipped muscle. Repeat MRI (Fig. 2) revealed adequate bone decompression of the posterior fossa and further

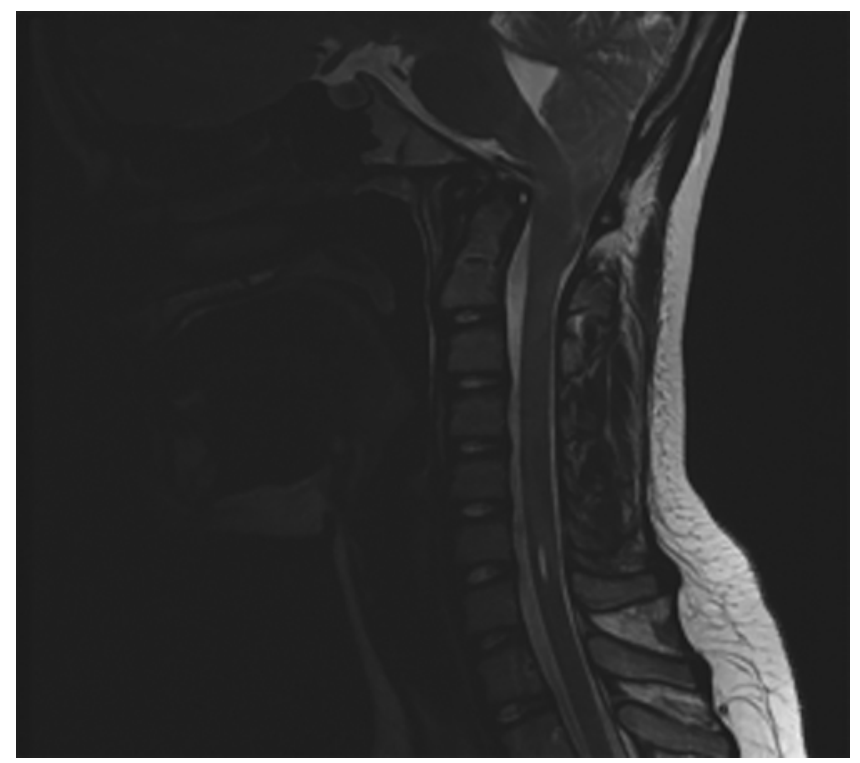

FIG. 1. Preoperative sagittal T2-weighted MR image showing the Chiari type 1.5 malformation with a small syrinx.

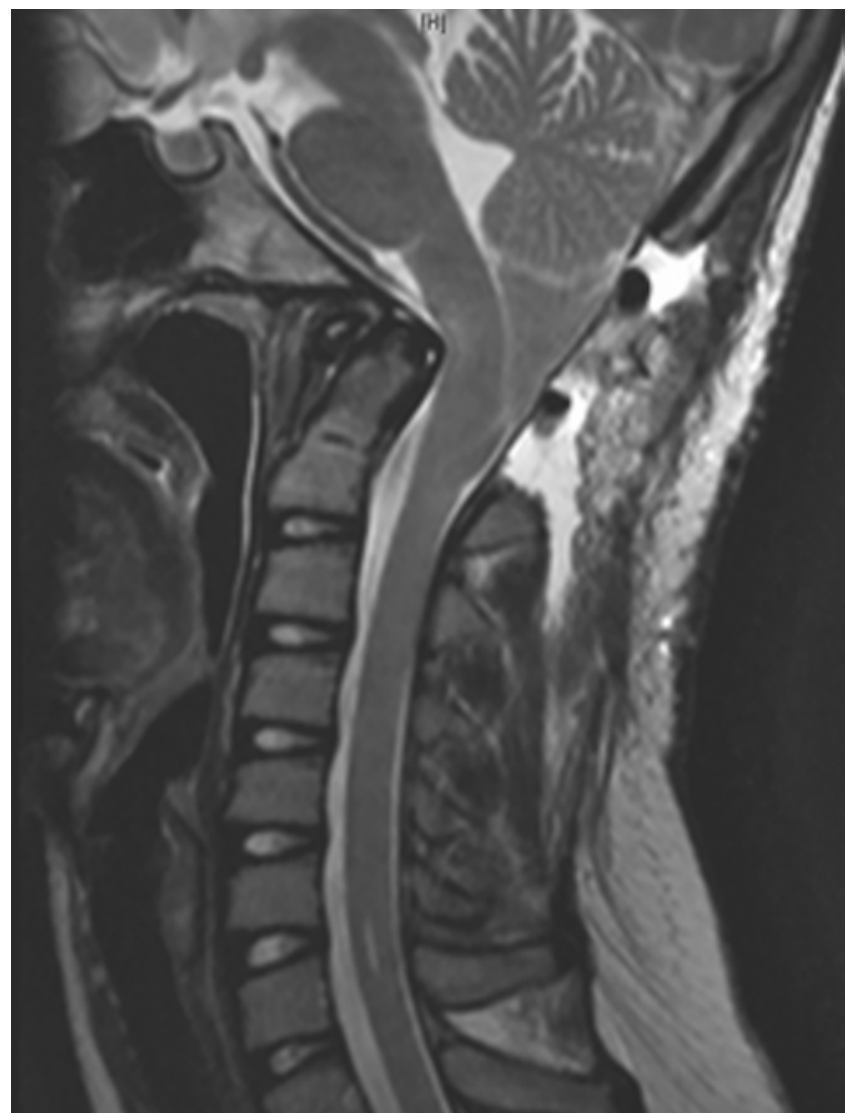

FIG. 2. Postoperative sagittal T2-weighted MR image showing the Chiari type 1.5 malformation with a small syrinx following posterior fossa decompression without dural opening.

neurosurgical intervention was not felt to be required at the time. We did note that in in one of the images (Fig. 2), there was a small remnant of the $\mathrm{C} 1$ posterior arch but we did not consider this atypical for the procedure, nor did we believe that the residual bone would hinder symptomatic improvement.

Two months later, examination demonstrated stable results, and a 7.0-mm right lateral rectus resection was performed. One day postoperatively she was orthophoric at distance and near, with only a trace adduction deficit of the left eye. Three weeks later she reported recurrent diplopia and examination showed an 18-PD esotropia at distance and an intermittent esotropia of 16 PDs at near, with full versions. Six weeks later the deviation worsened to 18 PDs at distance and 16 PDs at near, with full versions and a normal fundus bilaterally. The patient then underwent repeat posterior fossa decompression with dural opening and duraplasty. No additional bone was removed during repeat surgery. There were no unusual findings beyond tonsillar compression. It was thought that duraplasty provided more adequate decompression. One month postoperatively the esotropia improved to 10-12 PDs at distance and near with full versions. Two months later, she was diplopia free and had an esophoria of 2 PDs at distance and an exophoria of 1 PD at near. Ten months postoperatively her examination remained normal. Brain MRI showed ex- 


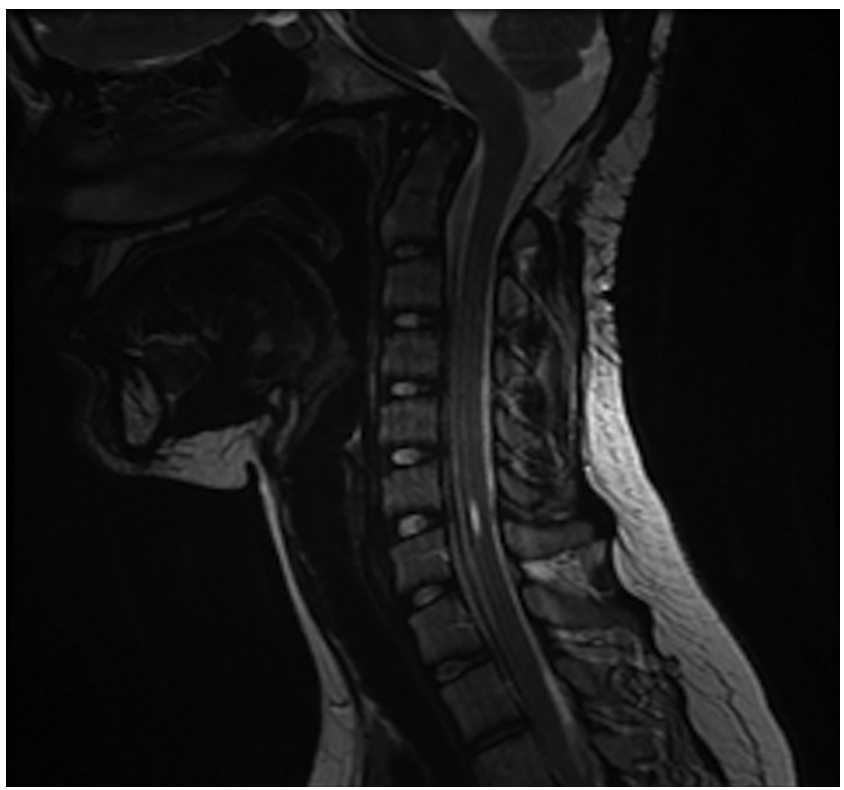

FIG. 3. Postoperative sagittal T2-weighted MR image showing the Chiari type 1.5 malformation with a small syrinx following posterior fossa decompression with dural opening and duraplasty.

cellent decompression with CSF surrounding the cerebellar tonsils and brainstem (Fig. 3). Follow-up one year later also demonstrated full ocular motility and orthophoria at distance and near.

\section{Discussion}

Esotropia with diplopia is not a common presentation of Chiari malformation. There are 16 reported cases of Chiari malformation presenting with esotropia. ${ }^{2,3,5,7,10,13 \text {, }}$ 16,20 The first case report of esotropia in a patient with Chiari malformation was published in $1984 .{ }^{16}$ In the case we present, the patient underwent initial strabismus surgery for esotropia with diplopia. The patient's symptoms initially improved, but within 2 weeks, the diplopia returned, and 3 weeks postoperatively the esotropia had worsened. The patient was orthophoric at near and had larger esotropia at distance. Larger esotropia at a distance is known as divergence insufficiency and is likely to be neurological in origin in the first 2 decades of life. Specifically, divergence insufficiency is thought to result from brainstem compression of the abducens nuclei or tegmentum in patients with Chiari malformation. Equal measurements or a larger deviation at near are less likely to be neurological in origin. The finding of larger esotropia at a distance suggested a neurological cause, and the patient underwent brain MRI that showed Chiari 1.5 malformation.

The patient underwent Chiari malformation decompression without dural opening. Symptoms initially improved, but several months later, the patient had worsening diplopia and esotropia requiring two further strabismus surgeries. Symptoms did not improve until the patient underwent repeat posterior fossa decompression with dural opening and duraplasty. We cannot say for certain but it is our view that the initial surgery did not afford an ad- equate decompression. It is assumed that the duraplasty was the limiting factor as the initial decompression was performed in a way that is typical for our patients who present with Chiari malformations and the patients symptoms improved with dural opening on repeat surgery.

In hindsight, we recommend suboccipital decompression and duraplasty in patients with extraocular movement dysfunction and significant Chiari malformation to ensure adequate outflow of CSF and to decrease compression. Case series from the past several decades suggest that abnormalities of eye movement in Arnold-Chiari malformation are due to compression of the herniating cerebellum against the caudal brainstem, and eye movements improve with posterior fossa decompression. ${ }^{11,17,18}$ Studies suggest that the sequence of treatment for comitant esotropia should be suboccipital decompression and then strabismus surgery if realignment does not occur. ${ }^{2}$ Studies suggest that Chiari malformation decompression alone can relieve the strabismus if done soon after onset of symptoms, before muscle length adaptation or higher cortical fusional disruptions occur. ${ }^{13,20}$ Vergence mechanisms are thought to originate in the mesencephalon, and the pathology of divergence insufficiency is thought to be secondary to compression of this area in Chiari malformation. ${ }^{2}$ The worsening diplopia and esotropia were not caused by a sixth cranial nerve palsy or a strabismus surgical complication because ductions or versions were always full in this patient. Furthermore, the patient had a skew deviation with vertical misalignment of the eyes that was not related to paresis of any of the cyclovertical muscles and was consistent with a posterior fossa lesion. ${ }^{4}$ Thus, early recognition of esotropia and proper classification can prevent worsening strabismus in patients with Chiari malformation. In special situations such as this, symptoms may need to be corrected by posterior fossa decompression with dural opening and duraplasty. Our intention in presenting this case is that this information may help guide providers who encounter similar problems in the future.

\section{References}

1. Aboulezz AO, Sartor K, Geyer CA, Gado MH: Position of cerebellar tonsils in the normal population and in patients with Chiari malformation: a quantitative approach with MR imaging. J Comput Assist Tomogr 9:1033-1036, 1985

2. Biousse V, Newman NJ, Petermann SH, Lambert SR: Isolated comitant esotropia and Chiari I malformation. Am J Ophthalmol 130:216-220, 2000

3. Bixenman WW, Laguna JF: Acquired esotropia as initial manifestation of Arnold-Chiari malformation. J Pediatr Ophthalmol Strabismus 24:83-86, 1987

4. Brodsky MC, Donahue SP, Vaphiades M, Brandt T: Skew deviation revisited. Surv Ophthalmol 51:105-128, 2006

5. Defoort-Dhellemmes S, Denion E, Arndt CF, BouvetDrumare I, Hache JC, Dhellemmes P: Resolution of acute acquired comitant esotropia after suboccipital decompression for Chiari I malformation. Am J Ophthalmol 133:723-725, 2002

6. Hernowo A, Eggenberger E: Skew deviation: clinical updates for ophthalmologists. Curr Opin Ophthalmol 25:485-487, 2014

7. Imes RK, Quinn TA: Acute comitant esotropia in Chiari 1 malformation. Ophthalmology 108:834, 2001

8. Jacobson DM: Divergence insufficiency revisited: natural 
history of idiopathic cases and neurologic associations. Arch Ophthalmol 118:1237-1241, 2000

9. Kowal L, Yahalom C, Shuey NH: Chiari 1 malformation presenting as strabismus. Binocul Vis Strabismus Q 21:18-26, 2006

10. Lewis AR, Kline LB, Sharpe JA: Acquired esotropia due to Arnold-Chiari I malformation. J Neuroophthalmol 16:4954, 1996

11. Malis LI, Cohen I, Gross SW: Arnold-Chiari malformation. AMA Arch Surg 63:783-798, 1951

12. Massey SL, Buland J, Hauber S, Piatt J Jr, Goraya J, Faerber E, et al: Acute VI nerve palsy in a 4 year-old girl with Chiari I malformation and pontomedullary extension of syringomyelia: case report and review of the literature. Eur J Paediatr Neurol 15:303-309, 2011

13. Passo M, Shults WT, Talbot T, Palmer EA: Acquired esotropia. A manifestation of Chiari I malformation. J Clin Neuroophthalmol 4:151-154, 1984

14. Paul KS, Lye RH, Strang FA, Dutton J: Arnold-Chiari malformation. Review of 71 cases. J Neurosurg 58:183-187, 1983

15. Pilon A, Rhee P, Newman T, Messner L: Bilateral abducens palsies and facial weakness as initial manifestations of a Chiari 1 malformation. Optom Vis Sci 84:936-940, 2007

16. Pokharel D, Siatkowski RM: Progressive cerebellar tonsillar herniation with recurrent divergence insufficiency esotropia. J AAPOS 8:286-287, 2004

17. Saez RJ, Onofrio BM, Yanagihara T: Experience with
Arnold-Chiari malformation, 1960 to 1970. J Neurosurg 45:416-422, 1976

18. Spooner JW, Baloh RW: Arnold-Chiari malformation: improvement in eye movements after surgical treatment. Brain 104:51-60, 1981

19. Steinbok P: Clinical features of Chiari I malformations. Childs Nerv Syst 20:329-331, 2004

20. Weeks CL, Hamed LM: Treatment of acute comitant esotropia in Chiari I malformation. Ophthalmology 106:23682371, 1999

\section{Disclosures}

The authors report no conflict of interest concerning the materials or methods used in this study or the findings specified in this paper.

\section{Author Contributions}

Conception and design: Strickland. Acquisition of data: Baker. Drafting the article: Strickland, Baker, Siatkowski. Critically revising the article: Mapstone, Siatkowski. Study supervision: Mapstone.

\section{Correspondence}

Timothy B. Mapstone: University of Oklahoma Health Sciences Center, Oklahoma City, OK. timothy-mapstone@ouhsc.edu. 